\title{
Retrospective Electrocardiography-Gated Real-Time Cardiac Cine MRI at 3T: Comparison with Conventional
} Segmented Cine MRI

\author{
Chen Cui, PhD ${ }^{1}$, Gang Yin, $B S^{1}$, Minjie Lu, $M D^{1}$, Xiuyu Chen, $M D^{1}$, Sainan Cheng, $M D^{1}$, Lu Li, MD', \\ Weipeng Yan, BS ${ }^{1}$, Yanyan Song, $\mathrm{PhD}^{1}$, Sanjay Prasad, $\mathrm{MD}^{2}$, Yan Zhang, $\mathrm{MD}^{1}$, Shihua Zhao, $\mathrm{MD}^{1}$
}

${ }^{1}$ Department of Magnetic Resonance Imaging, Fuwai Hospital, State Key Laboratory of Cardiovascular Disease, National Center for Cardiovascular Diseases, Peking Union Medical College and Chinese Academy of Medical Sciences, Beijing, China; ${ }^{2}$ Cardiovascular Magnetic Resonance Unit, Royal Brompton Hospital, Sydney Street, London, England

Objective: Segmented cardiac cine magnetic resonance imaging (MRI) is the gold standard for cardiac ventricular volumetric assessment. In patients with difficulty in breath-holding or arrhythmia, this technique may generate images with inadequate quality for diagnosis. Real-time cardiac cine MRI has been developed to address this limitation. We aimed to assess the performance of retrospective electrocardiography-gated real-time cine MRI at 3T for left ventricular (LV) volume and mass measurement. Materials and Methods: Fifty-one patients were consecutively enrolled. A series of short-axis cine images covering the entire left ventricle using both segmented and real-time balanced steady-state free precession cardiac cine MRI were obtained. End-diastolic volume (EDV), end-systolic volume (ESV), stroke volume (SV), ejection fraction (EF), and LV mass were measured. The agreement and correlation of the parameters were assessed. Additionally, image quality was evaluated using European CMR Registry (Euro-CMR) score and structure visibility rating.

Results: In patients without difficulty in breath-holding or arrhythmia, no significant difference was found in Euro-CMR score between the two techniques $(0.3 \pm 0.7$ vs. $0.3 \pm 0.5, p>0.05)$. Good agreements and correlations were found between the techniques for measuring EDV, ESV, EF, SV, and LV mass. In patients with difficulty in breath-holding or arrhythmia, segmented cine MRI had a significant higher Euro-CMR score $(2.3 \pm 1.2$ vs. $0.4 \pm 0.5, p<0.001)$.

Conclusion: Real-time cine MRI at 3T allowed the assessment of LV volume with high accuracy and showed a significantly better image quality compared to that of segmented cine MRI in patients with difficulty in breath-holding and arrhythmia. Keywords: Magnetic resonance imaging; Cardiac cine; Real-time acquisition

\section{INTRODUCTION}

Functional and volumetric assessment is important in the diagnosis and clinical management of cardiovascular diseases (1-3). Cardiac cine magnetic resonance imaging (MRI) is the current gold standard for noninvasive evaluation of ventricular morphological-functional parameters (4). Typically, cardiac cine MRI is obtained using a retrospective electrocardiography (ECG)-gated segmented sequence, in which segments of the entire $\mathrm{k}$-space are read out over multiple cardiac cycles. As such, each frame in segmented cine imaging has elements of several R-R intervals, and the image needs to be acquired during breath-hold to minimize respiratory motion artifacts.

Received April 19, 2018; accepted after revision August 15, 2018.

This study was supported by the Research Grant of National Natural Science Foundation of China (81620108015) and Capital Clinically Characteristic Applied Research Fund (Z161100000516110), Beijing, China.

Corresponding author: Shihua Zhao, MD, Department of Magnetic Resonance Imaging, Fuwai Hospital, State Key Laboratory of Cardiovascular Disease, National Center for Cardiovascular Diseases, Peking Union Medical College and Chinese Academy of Medical Sciences, No.167 North Lishi Road, Xicheng District, Beijing 100037, China.

- Tel: 86-10-88322539•Fax: 86-10-88398866•E-mail: cjrzhaoshihua2009@163.com

This is an Open Access article distributed under the terms of the Creative Commons Attribution Non-Commercial License (https:// creativecommons.org/licenses/by-nc/4.0) which permits unrestricted non-commercial use, distribution, and reproduction in any medium, provided the original work is properly cited. 
Multiple short axis cine scans are required to ensure full myocardial coverage and can often take 5-15 minutes. For patients in high New York Heart Association classes, voluntary breath-holds are difficult to perform to generate images with optimal image quality. Similarly, in patients with cardiac arrhythmia, image degradation can occur because of motion artifact, thus affecting image quality. Real-time cardiac cine MRI has been developed to address these limitations, in which the k-space of all temporal phases in one slice can be filled up in a single R-R interval and the cine images covering the entire left ventricle can be acquired in one breath-hold (5). Because of the "realtime" nature of this technique, artifacts associated with respiratory motion and arrhythmia can be reduced.

Real-time cine sequence has been validated at 1.5 (68 ) and 3 tesla $(T)(9,10)$ for volumetric assessment with different acceleration techniques including compressed sensing (CS) $(10,11)$ and temporal sensitivity encoding (12). However, most of the reported real-time sequences were implemented with prospective ECG triggering for data acquisition $(6,10,13)$, which did not cover the entire cardiac cycle and will lead to underestimation of enddiastolic volume (EDV) $(11,13)$. Other techniques can be used to obtain cardiac cine covering the entire R-R interval by retrospective reconstruction of data acquired over multiple cardiac cycles $(14,15)$. However, in patients with arrhythmia, additional manual work is required to discard dissimilar heartbeats (15). In addition, 80-120 seconds are required to reconstruct a single slice of cardiac cine (14, $15)$ in these techniques. In order to address this real-world clinical limitation, in the present study, we aimed to assess the performance of real-time cine imaging sequence at $3 \mathrm{~T}$ in patients for assessment of left ventricular (LV) functional parameters.

We hypothesized that real-time cine with single-shot acquisition (to generate cardiac cine with data acquired in a single heartbeat) using retrospective gating can generate images with sufficient quality for robust volumetric assessment that overcomes limitations due to underlying arrhythmia as well as suboptimal breath-holding.

\section{MATERIALS AND METHODS}

\section{Study Population}

The protocol of this prospective study was approved by the Institutional Review Board in our hospital, and all the patients gave informed consent in accordance with the
Declaration of Helsinki. Clinical patients with a range of cardiac conditions confirmed by echocardiography and 12lead ECG were consecutively enrolled from June to August 2016. Fifty-one patients were referred for MRI examination, and cardiac cine MRI was a part of the conventional MRI protocol. Three patients for whom MRI was contraindicated were excluded.

Difficulty in breath-holding was considered to be present when an unstable respiratory curve was observed on the physiology display panel in the user interface of the scanner. The patients were divided into two groups: group 1 $(n=33)$, patients without arrhythmia or imperfect breathholding; and group $2(n=15)$, patients with arrhythmia and/or difficulty in breath-holding.

\section{MRI Scan Protocol}

MRI scan was performed on a clinical 3T magnetic resonance (MR) scanner (Ingenia, Philips Healthcare, Best, the Netherland) with a high-performance gradient system (45 mT/m; maximum slew rate, $200 \mathrm{mT} / \mathrm{m} / \mathrm{ms}$ ). Patients were scanned in the supine position using a 32-channel phased-array dStream Torso coil (Philips Healthcare). MRcompatible ECG electrodes and leads were used to obtain an ECG signal for cardiac synchronization. MRI were acquired during breath-holding in end expiration. Transverse, sagittal, and coronal scout images were obtained. Subsequently, a series of standard LV short-axis cines were acquired using the segmented balanced steady-state free precession (b-SSFP) sequence with retrospective ECG gating in a stack of 8 contiguous slices spanning the entire left ventricle from the base to apex. Typical imaging parameters are presented in Table 1.

The retrospective ECG-gated real-time cardiac cine MRI in our study was based on the b-SSFP sequence. Retrospective ECG-gating was used to cover the entire cardiac cycle with 15 phases. In addition, view-sharing was set to $50 \%$ to improve the temporal resolution. The scan time for realtime cardiac cine MRI was equal to two heartbeats. One heartbeat was used to obtain magnetization steady state, and the other heartbeat was used for data acquisition (Fig. 1). The real-time cine MRI was performed in an identical orientation with the same slice thickness and interslice gap as those used for the segmented cine sequence. The detailed parameters of real-time cine are presented in Table 1. No offline reconstruction was required to generate the real-time cine MRI. The two sets of LV cardiac cines were acquired in random order. In addition, the imaging 
Table 1. Detailed Parameters of Sequences

\begin{tabular}{lcc}
\hline \multicolumn{1}{c}{ Parameters } & Segmented Cine & Real-Time Cine \\
\hline Sequence type & 2D cine b-SSFP & 2D cine b-SSFP \\
TR/TE & $2.8 / 1.4$ & $2.6 / 1.3$ \\
FOV & $350 \times 322$ & $350 \times 322$ \\
In plane resolution (mm)* & $1.6 \times 1.8$ & $2.8 \times 3.0-4.2$ \\
Temporal resolution (ms)* & $30-45$ & $40-60$ \\
View-sharing $(\%)$ & - & 50 \\
Phases & 25 & 15 \\
Thickness (mm) & 8 & 8 \\
Interslice gap (mm) ${ }^{\dagger}$ & $0-3$ & $0-3$ \\
Flip angle $\left({ }^{\circ}\right)$ & 45 & 45 \\
Bandwidth $(\mathrm{Hz})$ & 1900 & 2700 \\
SENSE factor & 1.5 & 2 \\
Breath-holds & 8 & 1 \\
ECG mode & Retrospective & Retrospective \\
\hline
\end{tabular}

*Parameters varied depending on $\mathrm{HR}$, †Parameter varied depending on size of heart. b-SSFP = balanced steady-state free precession, $E C G$ = electrocardiography, FOV = field of view, HR = heart rate, SENSE $=$ sensitivity encoding,$T E=$ echo time, $T R=$ repetition time, $2 \mathrm{D}=$ two-dimensional

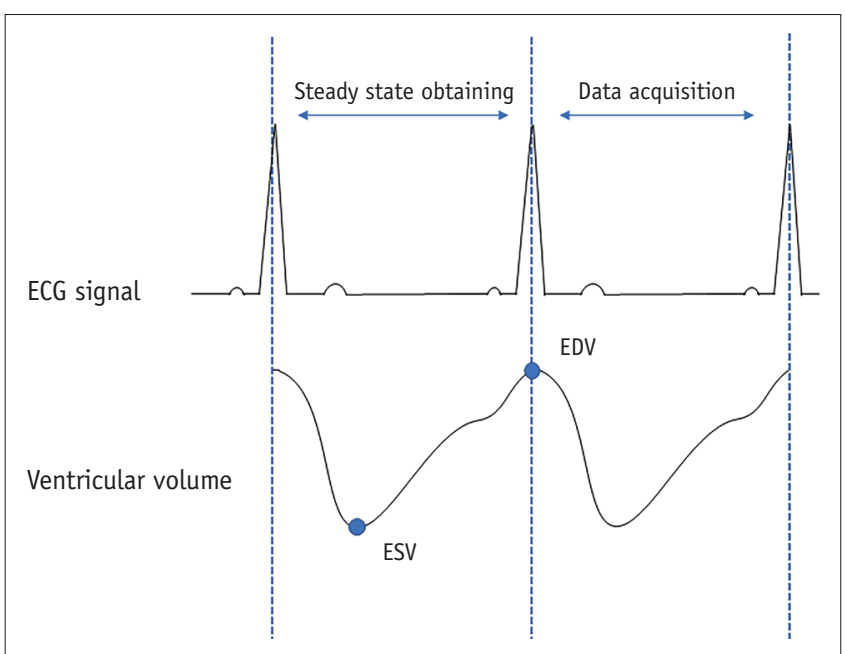

Fig. 1. Schematic diagram for data acquisition in retrospective gated real-time cardiac cine. ECG = electrocardiography, EDV = enddiastolic volume, ESV = end-systolic volume

parameters permitted the specific absorption rate to be within the regulatory limits for all the sequences in the present study.

\section{Cardiac Volumetric and Mass Analysis}

The EDV, end-systolic volume (ESV), stroke volume (SV), ejection fraction (EF), and LV mass were measured using the software of CVI 42 (Circle Cardiovascular Imaging Inc., Calgary, Canada). All the short-axis cine images (including both segmented cine and real-time cine images) were analyzed in a randomized and blinded manner. Briefly, the endo- and epicardial contours were automatically detected with manual correction. Papillary muscles and myocardial trabeculations were included in the ventricular cavity volume. End-diastole and end-systole was defined as the phase of the cardiac cycle with the largest and smallest ventricular cavities, respectively (16). A functional analysis of all participants was performed by a cardiovascular radiologist with 7 years of experience. To test intraobserver variability, the functional analysis was repeated by the same radiologist in a subset of 15 randomly selected participants two months later. The interobserver variability was also tested in the same subset of participants by another radiologist with 5 years of experience.

\section{Image Quality Analysis}

The image quality of cardiac cine MRI was analyzed according to two independent criteria, European CMR Registry (Euro-CMR) score $(17,18)$ and structure visibility rating based on 4-point scales (19). The former focused on the artifacts, whereas the latter emphasized the visibility of cardiac structures. A radiologist with 7 years of experience in cardiac MRI performed all the quality analysis.

The Euro-CMR score was defined as follows: 1 point was given if any artifacts (wrap around, respiratory ghost, cardiac ghost, image blurring/mis-triggering, metallic, or shimming) impeded the visualization of more than onethird of the LV endocardial border at end-systole or enddiastole on a single slice. According to the number of slices that were involved, 1-3 points were given (17).

For structure visibility rating, the reviewers were asked to rate the following cardiac features: endocardial border definition, epicardial border definition, papillary muscle visualization, myocardium visualization, blood pool contrast, and cardiac motion. According to the visibility of the structure, images were rated on a scale as follows: $4=$ excellent, $3=$ good, $2=$ poor, and $1=$ no visibility.

\section{Statistical Analysis}

All statistical analyses were performed using the MedCalc software (version 16.2.0, MedCalc Software bvba, Ostend, Belgium). Normality of the data was tested using the Shapiro-Wilk test and quantile-quantile plots. LV functional parameters and image quality ratings derived from segmented and real-time cines were compared using paired $t$ test or nonparametric Wilcoxon matched pair test depending on whether or not the data were normally distributed. Simple linear regression and Bland-Altman 
analysis were performed to assess the correlation and agreement of the functional parameters assessed by the two methods. A $p$ value of 0.05 or less was considered to indicate a statistically significant difference.

\section{RESULTS}

\section{Patient Characteristics}

Fifty-one patients were consecutively enrolled. Three patients were excluded because of claustrophobia, and images were acquired successfully in the remaining 48 patients. The average scan time of segmented cine for each slice was $7.92 \pm 2.15$ seconds. The mean scan time of segmented cine MRI for the 8 slices of short-axis stack was significantly longer than that of the real-time cine (168.9 \pm 7.8 seconds for segmented cine vs. $10.8 \pm 1.2$ seconds for real-time cine, $p<0.001$ ).

The demographic data are summarized in Table 2. The mean age of the participants was 47 years, and $83.3 \%$ of the patients were male. Hypertrophic cardiomyopathy was the main reason for performing cardiac MRI. In addition, the mean heart rate $(H R)$ of the patients was $67 \pm 11$ beats/min (range: $50-88$ beats/min).

of the 48 patients, 9 had a history of arrhythmia and had detectable arrhythmia on the ECG display panel during the MRI scan; 3 patients had difficulty in breath-holding and another 3 patients had both arrhythmia and difficulty in breath-holding. Of the 12 patients with arrhythmia, 3 had persistent atrial fibrillation, 5 had paroxysmal atrial fibrillation, and 4 had frequent ventricular premature beats. According to the predefined criteria, 33 patients were classified into group 1 and 15 were classified into group 2. The representative images for the both groups are shown in Figures 2 and 3.

\section{Image Quality Assessment}

Detailed Euro-CMR score for segmented and real-time cine MRI are summarized in Table 3. In group 1, images acquired by both sequences had a low Euro-CMR score, and minor image artifacts affected the identification of the endo- and epicardial contours in LV volume and mass measurements. Wilcoxon matched pair test showed no significant difference in the averaged Euro-CMR score between the techniques (Euro-CMR score: $0.3 \pm 0.7$ for segmented cine vs. $0.3 \pm$ 0.5 for real-time cine, Wilcoxon $p=0.903)$. In group 2, segmented cine had a significantly higher Euro-CMR score (Euro-CMR score: $2.3 \pm 1.2$ for segmented vs. $0.4 \pm 0.5$ for real-time, Wilcoxon $p$ value $<0.001)$. Mis-triggering due to

Table 2. Patients' Characteristics

\begin{tabular}{|c|c|c|c|}
\hline & All $(n=48)$ & Group $1(n=33)$ & Group $2(n=15)$ \\
\hline Age, years & $47 \pm 16$ & $43.1 \pm 14.1$ & $54.1 \pm 15.0$ \\
\hline Male $(\%)$ & $40(83.3)$ & $27(81.8)$ & $13(86.7)$ \\
\hline BMI $\left(\mathrm{kg} / \mathrm{m}^{2}\right)$ & $25.66 \pm 4.67$ & $26.76 \pm 4.70$ & $23.24 \pm 4.02$ \\
\hline $\operatorname{BSA}\left(m^{2}\right)$ & $2.96 \pm 0.29$ & $2.99 \pm 0.30$ & $2.90 \pm 0.27$ \\
\hline $\mathrm{HR}$, beats/min & $67 \pm 11$ & $64 \pm 11$ & $72 \pm 12$ \\
\hline \multicolumn{4}{|l|}{ Cardiovascular risk factors (\%) } \\
\hline Hypertension & $12(25)$ & $11(33.3)$ & $1(6.7)$ \\
\hline Hypercholesterolemia & $12(25)$ & $9(27.27)$ & $3(20)$ \\
\hline Diabetes & $2(4.1)$ & $1(3.03)$ & $1(6.67)$ \\
\hline Smoking & $11(22.9)$ & $6(18.2)$ & $5(33.3)$ \\
\hline \multicolumn{4}{|l|}{ Diagnosis (\%) } \\
\hline Coronary artery disease & $7(14.5)$ & $5(15.2)$ & $2(13.3)$ \\
\hline Dilated cardiomyopathy & $4(8.3)$ & $3(9.1)$ & $1(6.7)$ \\
\hline Hypertrophic cardiomyopathy & $19(39.6)$ & $13(39.3)$ & $6(40)$ \\
\hline Valvular disease & $3(6.3)$ & $2(6.1)$ & $1(6.7)$ \\
\hline Other & $15(31.2)$ & $10(30.3)$ & $4(26.7)$ \\
\hline \multicolumn{4}{|l|}{ Arrhythmia (\%) } \\
\hline Persistent atrial fibrillation & $3(6.3)$ & - & $3(20)$ \\
\hline Paroxysmal atrial fibrillation & $6(12.5)$ & - & $6(40)$ \\
\hline Ventricular premature beats & $4(8.3)$ & - & $4(26.7)$ \\
\hline Difficulty in breath-holding & $3(6.3)$ & - & $3(20)$ \\
\hline
\end{tabular}

Group 1: patients without arrhythmia or difficulty in breath-holding, Group 2: patients with arrhythmia and/or difficulty in breathholding. BMI = body mass index, BSA = body surface area 


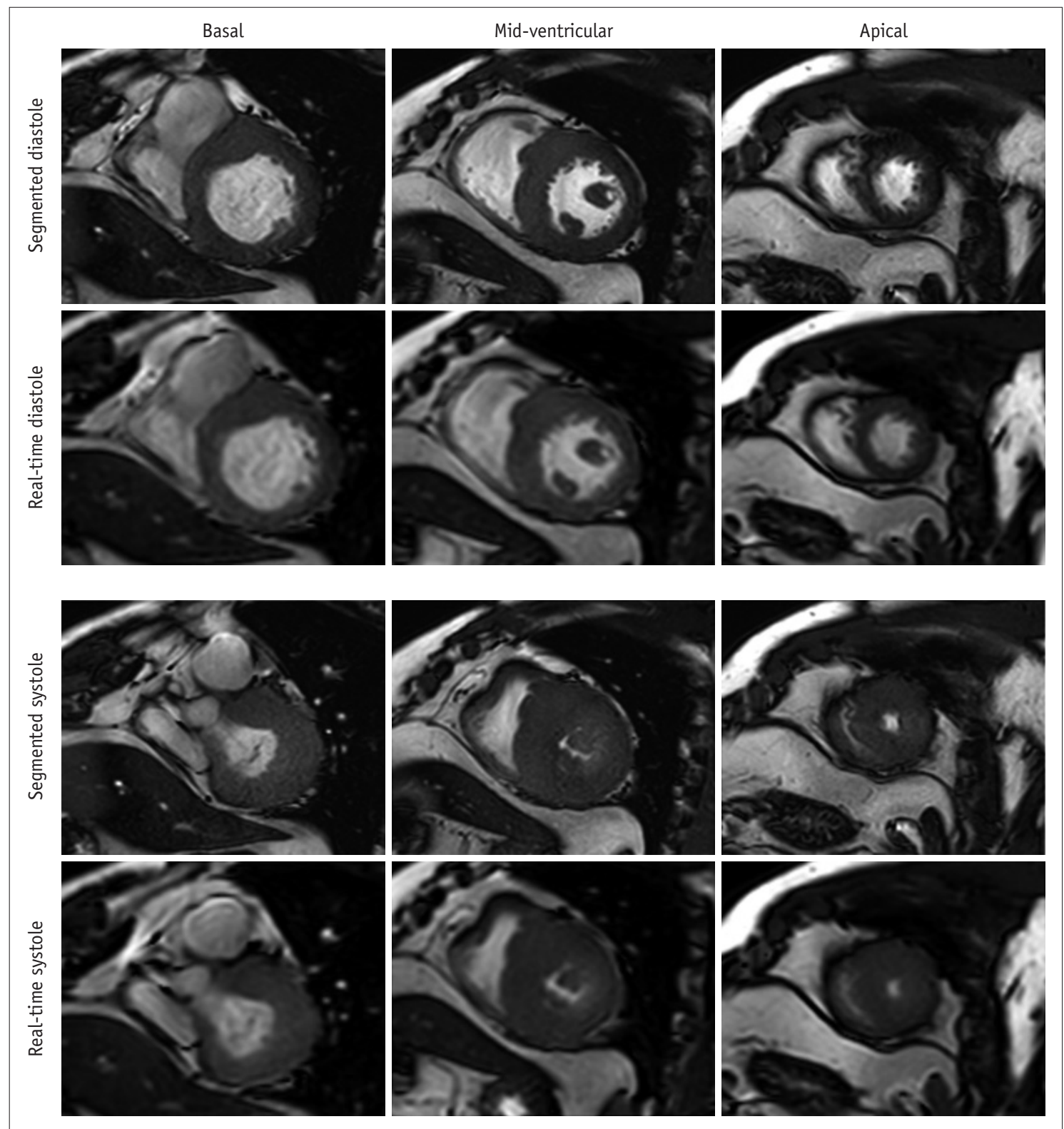

Fig. 2. Representative images of patients without difficulty in breath-holding and arrhythmia. Short-axis view images in enddiastole and end-systole phases in hypertrophic cardiomyopathy patient with heart rate of 59 beats/min.

arrhythmia was the most frequent factor found to influence the identification of myocardium contours.

Results of cardiac structure visibility rating are summarized in Table 4 and Figure 4. In group 1, all the segmented cine images and most of the real-time cine images were rated as 3 or 4 , except for 3 sets of realtime cines, which got a rating of 2 in endocardial border definition, papillary muscle visualization, and blood pool contrast, respectively. Image reviewers strongly preferred the segmented cines for blood pool contrast and endocardial border definition, papillary muscle visualization, and myocardium visualization (all Wilcoxon paired $p$ values $<0.05)$. In addition, the reviewers reported that the visualization of cardiac motion of real-time cines was slightly influenced by the lower effective temporal resolution.

With regard to group 2, the visualization of endocardial and epicardial border, papillary muscle, and myocardium in segmented cine MRI was significantly impaired by mistriggering or respiratory artifacts, compared with real-time cine (all Wilcoxon paired $p$ values $<0.05$ ). The visualization of cardiac motion was impeded in segmented cine as well (mean rating: $3.0 \pm 0.7$ for segmented cine vs. $3.6 \pm 0.5$ for real-time cine, Wilcoxon paired $p<0.05)$. The blood 




Fig. 3. Representative images of patients with difficulty in breath-holding and arrhythmia. Short-axis view images in end-diastole and end-systole phases in patient with atrial fibrillation. In contrast to real-time cardiac cine, endocardial contour was difficult to identify in conventional segmented cine in mid-ventricular and apical slices.

Table 3. Euro-CMR Scores for Image Acquired by Segmented Cine and Those by Real-Time Cine

\begin{tabular}{|c|c|c|c|c|c|c|c|c|}
\hline & \multicolumn{4}{|c|}{ Segmented Cine (Group 1, $\mathrm{n}=33$ /Group 2, $\mathrm{n}=15$ ) } & \multicolumn{4}{|c|}{ Real-Time Cine (Group 1, $\mathrm{n}=33 /$ Group 2, $\mathrm{n}=15$ ) } \\
\hline & 0 & 1 & 2 & 3 & 0 & 1 & 2 & 3 \\
\hline Wrap-around & $33 / 15$ & $-/-$ & $-/-$ & $-/-$ & $33 / 15$ & $-/-$ & $-/-$ & $-/-$ \\
\hline Respiratory ghost & $33 / 10$ & $-/ 4$ & $-/-$ & $-/ 1$ & $33 / 15$ & $-/-$ & $-/-$ & $-/-$ \\
\hline Cardiac ghost & $33 / 15$ & $-/-$ & $-/-$ & $-/-$ & $24 / 11$ & $8 / 4$ & $1 /-$ & $-/-$ \\
\hline Blurring/mis-triggering & $33 / 3$ & $-/ 3$ & $-/ 5$ & $-/ 4$ & $33 / 14$ & $-/ 1$ & $-/-$ & $-/-$ \\
\hline Metallic artifact & $33 / 15$ & $-/-$ & $-/-$ & $-/-$ & $33 / 15$ & $-/-$ & $-/-$ & $-/-$ \\
\hline Shimming artifact & $28 / 14$ & $3 /-$ & $1 / 1$ & $1 /-$ & $33 / 15$ & $-/-$ & $-/-$ & $-/-$ \\
\hline Total score, median (IQR) & \multicolumn{4}{|c|}{$0.3 \pm 0.7 ; 0(0,0) / 2.3 \pm 1.2 ; 2(1,3)$} & \multicolumn{4}{|c|}{$0.3 \pm 0.5 ; 0(0,1) / 0.4 \pm 0.5 ; 0(0,0)$} \\
\hline
\end{tabular}

Group 1, patients without arrhythmia or difficulty in breath-holding; Group 2, patients with arrhythmia and/or difficulty in breathholding. Euro-CMR = European CMR Registry, IQR = interquartile range 
Table 4. Structure Visualization Rating for Images Acquired by Segmented and Those by Real-Time Cine

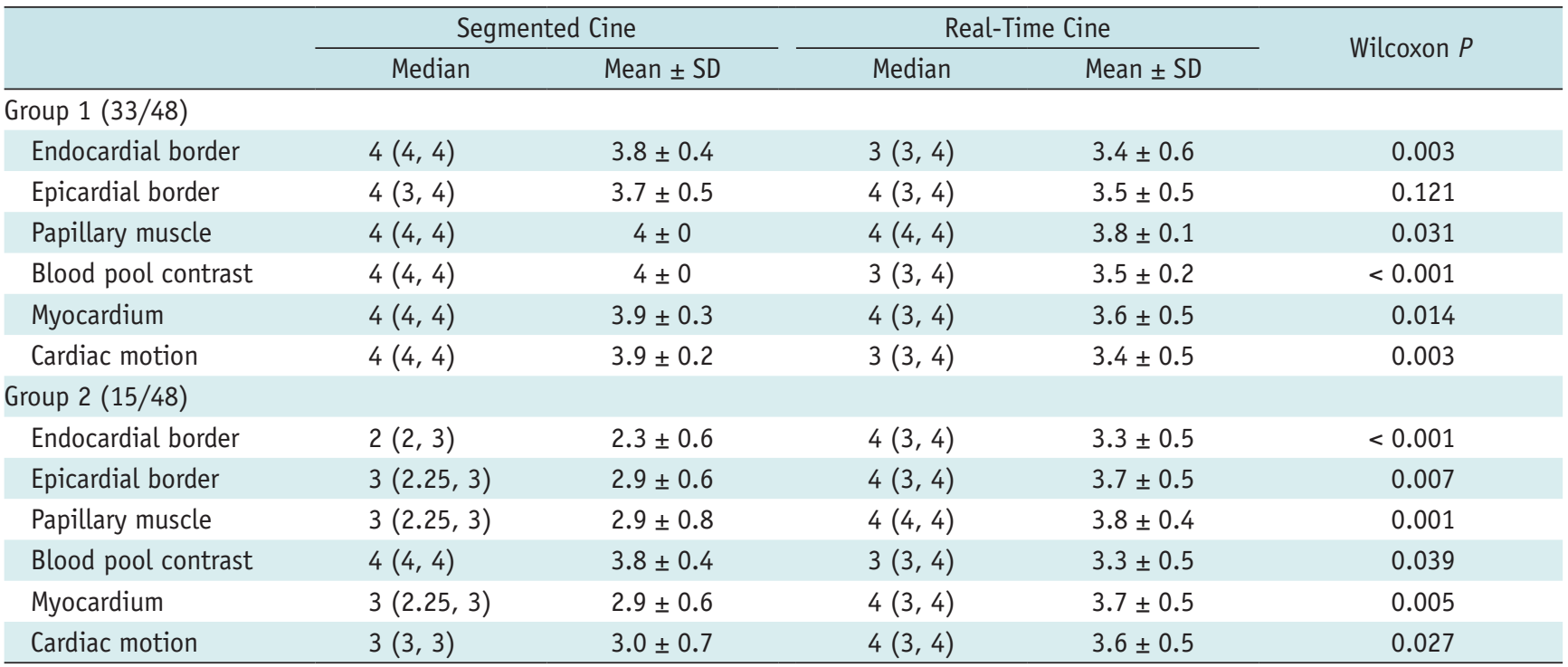

Group 1, patients without arrhythmia or difficulty in breath-holding; Group 2, patients with arrhythmia and/or difficulty in breathholding. SD = standard deviation

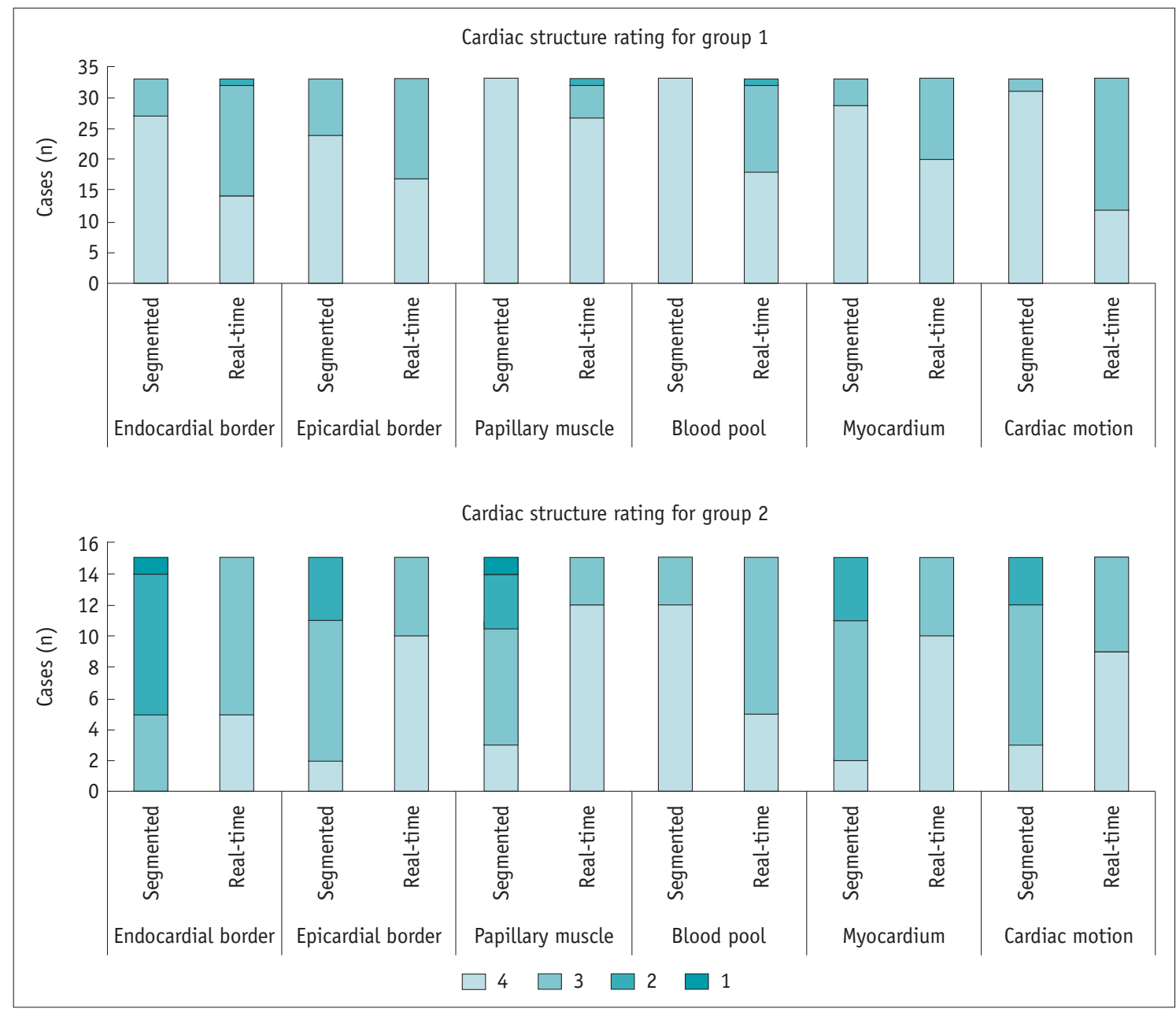

Fig. 4. Reviewer rating of endocardial border definition, epicardial border definition, papillary muscle visualization, myocardium visualization, blood pool contrast, and cardiac motion for real-time and segmented cine in patients from both groups. 
Table 5. LV Volume Measurements for Patients in Group 1

\begin{tabular}{lccc}
\hline & Segmented Cine & Real-Time Cine & $P$ \\
\hline LVEDV $(\mathrm{mL})$ & $160.7 \pm 59.9$ & $160.8 \pm 61.5$ & 0.929 \\
LVESV $(\mathrm{mL})$ & $87.4 \pm 63.2$ & $88.2 \pm 64.4$ & 0.674 \\
LVSV $(\mathrm{mL})$ & $73.3 \pm 19.2$ & $72.6 \pm 20.1$ & 0.495 \\
LV mass $(\mathrm{g})$ & $178.2 \pm 76.3$ & $180.8 \pm 77.0$ & 0.086 \\
LVEF $(\%)$ & $50.1 \pm 16.6$ & $49.1 \pm 16.8$ & 0.406
\end{tabular}

$\mathrm{EDV}=$ end-diastolic volume, $\mathrm{EF}=$ ejection fraction, $\mathrm{ESV}=$ endsystolic volume, LV = left ventricular, SV = stroke volume

pool contrast in segmented cine was better than that in real-time cine $(3.8 \pm 0.4$ for segmented cine vs. 3.3 \pm 0.5 for real-time cine, Wilcoxon paired $p<0.05$ ). It is noteworthy that in segmented cine, 10 and 4 patients got a rating lower than 3 for endocardial and epicardial border definition, respectively. No structure was rated lower than 3 in the real-time cine.

\section{Volumetric Parameters}

The functional and mass measurements were successfully performed in group $1(n=33)$. There were no significant differences in LV measurements between the two methods (Table 5). Using segmented cine as the standard reference, Bland-Altman analysis showed good agreements between the two methods for measuring the EDV (limits of agreement $=-0.1 \pm 7.3 \mathrm{~mL}$ ), ESV (limits of agreement $=$ $-0.8 \pm 5.0 \mathrm{~mL}$ ), EF (limits of agreement $=0.4 \pm 2.7 \%$ ), SV (limits of agreement $=-0.7 \pm 5.8 \mathrm{~mL}$ ), and LV mass (limits of agreement $=-3.1 \pm 10.7$ ). The Bland-Altman plots were illustrated in Figure 5. The linear regression yielded good correlations between the two methods in EDV $(y=5.118+$ $\left.0.968 x, R^{2}=0.973, p<0.001\right)$, ESV $\left(y=1.154+0.978 x, R^{2}\right.$ $=0.994, p<0.001), E F\left(y=1.762+0.972 x, R^{2}=0.969, p\right.$ $<0.001)$, SV $\left(y=7.038+0.912 x, R^{2}=0.917, p<0.001\right)$, and $L V$ mass $\left(y=7.732+0.940 x, R^{2}=0.986, p<0.001\right)$ as shown in Figure 6.

\section{DISCUSSION}

With the increasing clinical use and benefit of cardiac MRI, an important challenge is delivering robust analysis of LV volumetric assessment for the accruing population of patients with underlying arrhythmia or difficulty with breath-holding. Our study showed that retrospective ECGgated real-time cine MRI at 3T had performance equivalent to that of the current gold standard for LV volumetric and mass measurements in patients with good breath-holding capability and sinus rhythm. Moreover, for patients with arrhythmia and/or those who could not perform breathholding, this sequence provided better visualization of cardiac structures with fewer artifacts in comparison with the conventional segmented cardiac cines.

The image quality of cardiac cine is compromised in a subgroup of patients having arrhythmia and difficulty in breath-holding. According to previous studies, the prevalence of atrial fibrillation is about $18 \%$ in patients with hypertrophic cardiomyopathy (20) and reaches $12 \%$ in cardiac patients aged 60 years or more (21). In addition, dyspnea and orthopnea are the major symptoms of heart failure (22). In these patients, contour detection in volumetric analysis is difficult to perform because of motion artifacts. To overcome the limitation of the segmented cine, real-time cardiac cine with prospective ECG triggering can be used as an alternative solution. This technique is less sensitive to imperfect breath-holding and arrhythmia but has the potential to underestimate the $\operatorname{EDV}(11,13)$, because the "trigger window" between the end of data sampling and the next expected $R$ wave accounts for 10$15 \%$ of the R-R interval. Multiple heartbeats can be used in prospective real-time cine to cover the full cardiac cycle $(6,23)$. The performance of these techniques has not been validated in patients with arrhythmia. Another approach to capture the entire cardiac cycle is to retrospectively reconstruct the cardiac cine using data acquired from multiple heartbeats $(14,15)$. Implemented with motion correction, these sequences can be performed during free breathing. However, for patients with severe arrhythmia, additional work is required to generate cardiac cines (15) and the reconstruction time is relatively long (80-120 seconds) for one slice (14). In contrast, the average scan time for covering the entire left ventricle for the real-time cine used in the present study was $10.8 \pm 1.2$ seconds; thus, the approach used in this study drastically reduces the scan time and can optimize the cardiac MRI workflow.

In comparison with segmented cardiac cine, one of the limitations of the real-time cine is the low spatial resolution. The good agreements and correlations in the present study indicated that a relatively low spatial resolution did not affect the accuracy of volumetric assessment at 3T. As reported by Yamamuro et al. (12), temporal resolution is more important than spatial resolution in ventricular functional evaluation. The temporal resolution of the real-time sequence in the present study was no more than $60 \mathrm{~ms}$, which is close to the isovolumetric contraction at end-diastole and end-systole (about $50 \mathrm{~ms}$ 

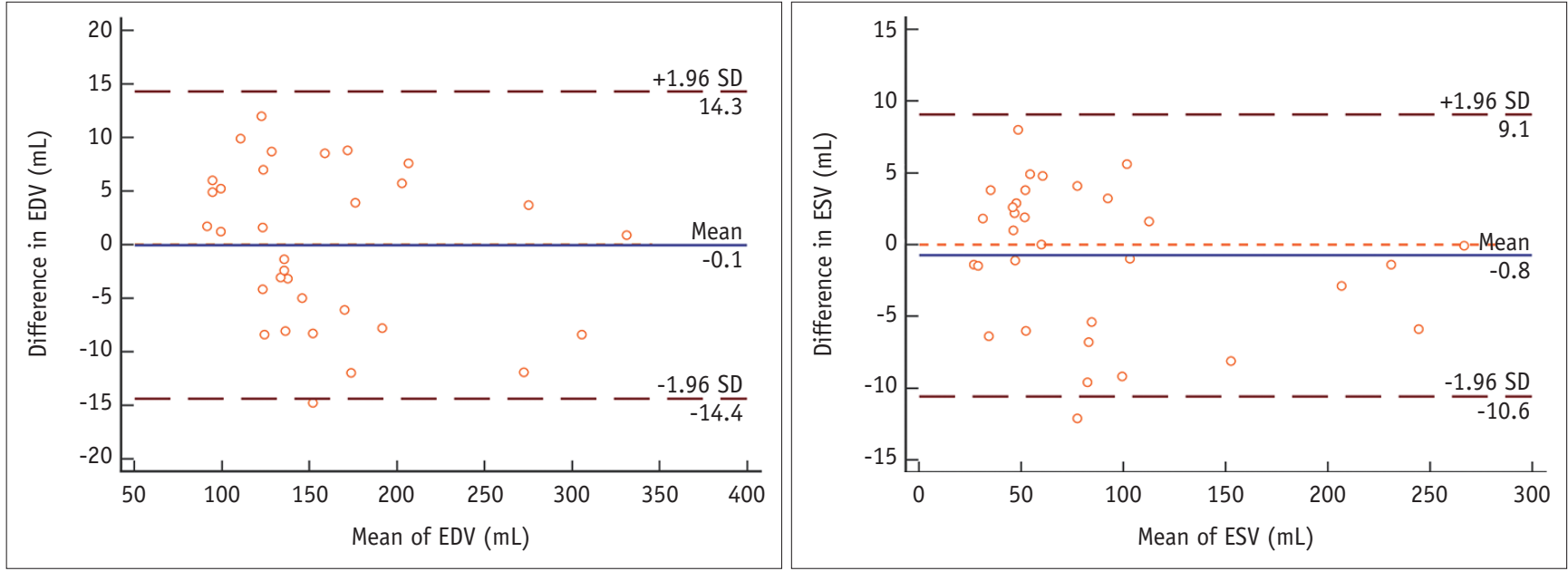

A

B
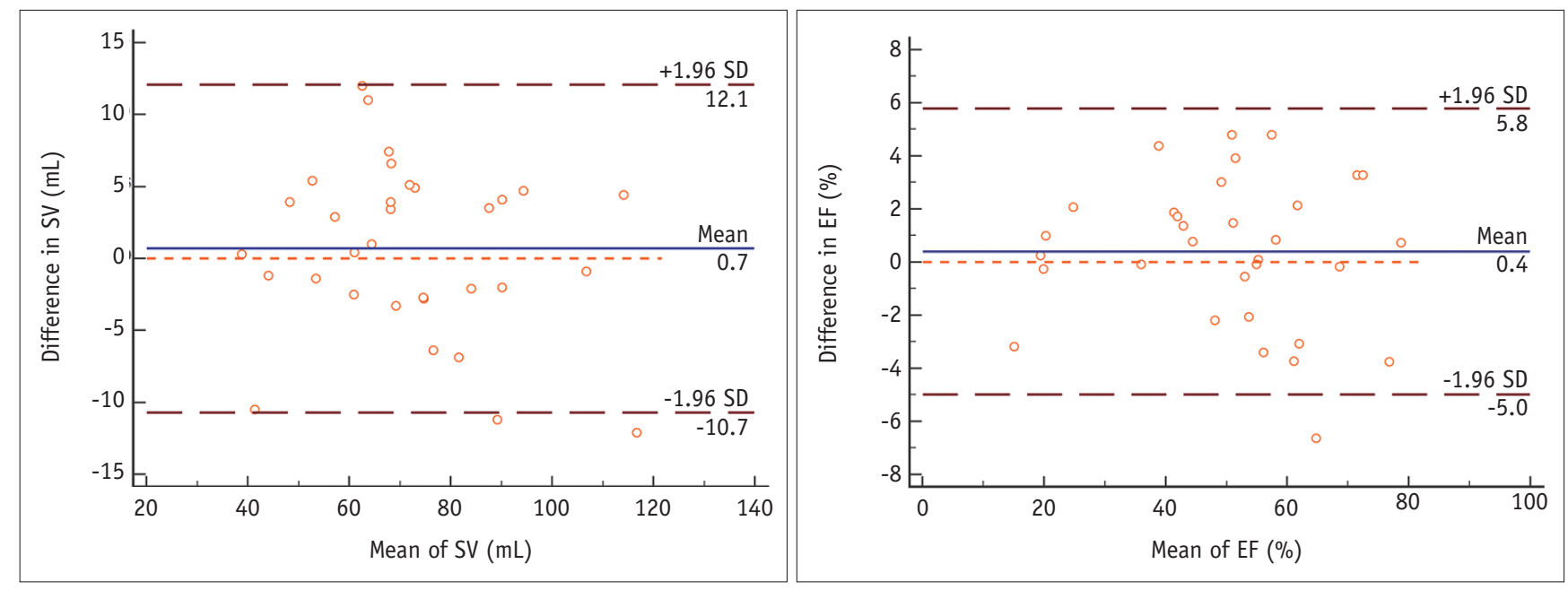

C

D

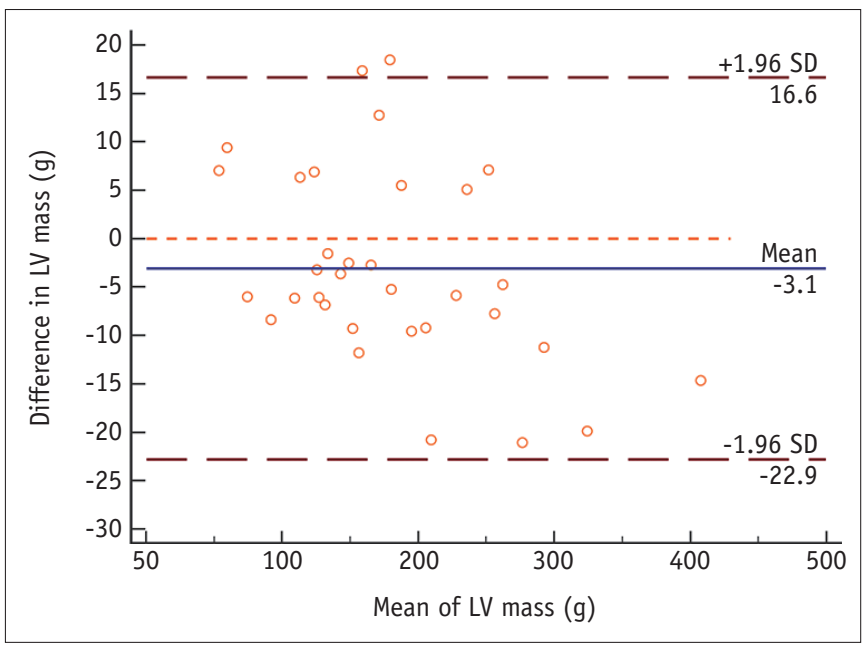

E

Fig. 5. Bland-Altman analysis shows good agreements in measurements of EDV (A), ESV (B), SV (C), EF (D) and LV mass (E). Blue line indicates mean value and brown dashed line indicates $95 \%$ confidence interval. $E F=$ ejection fraction, $L V=$ left ventricular, $S D=s t a n d a r d$ deviation, SV = stroke volume 

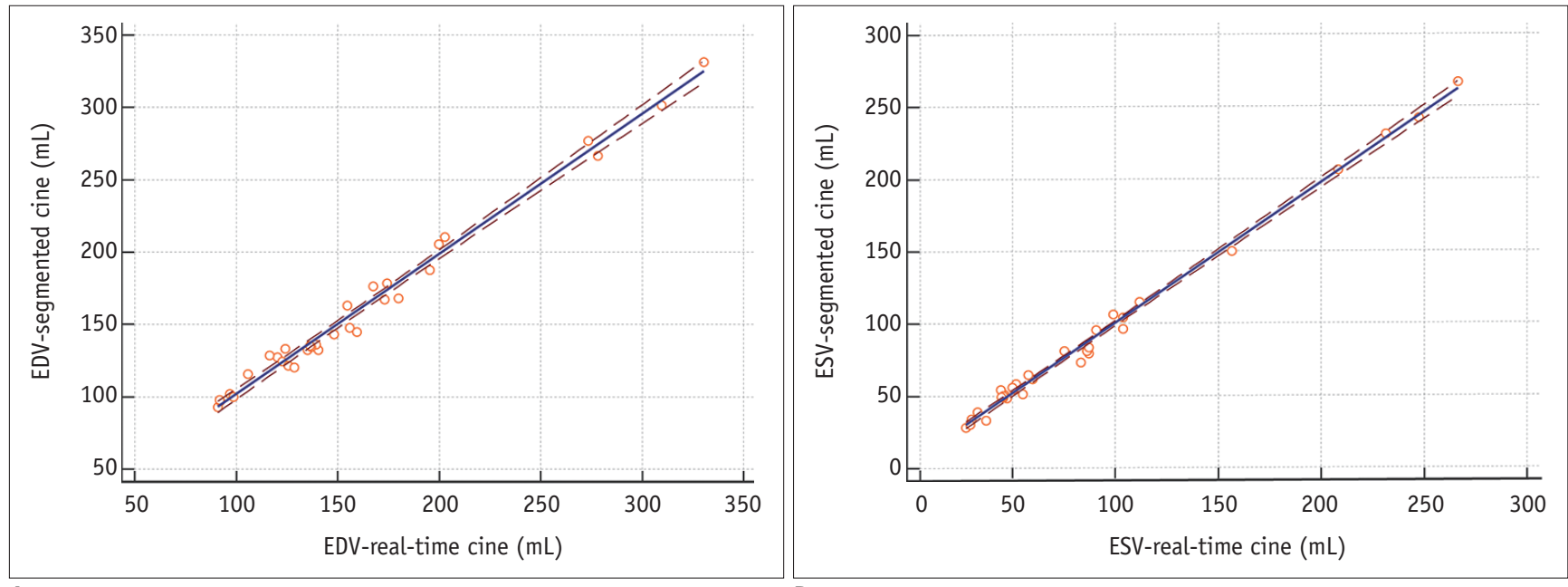

A

B
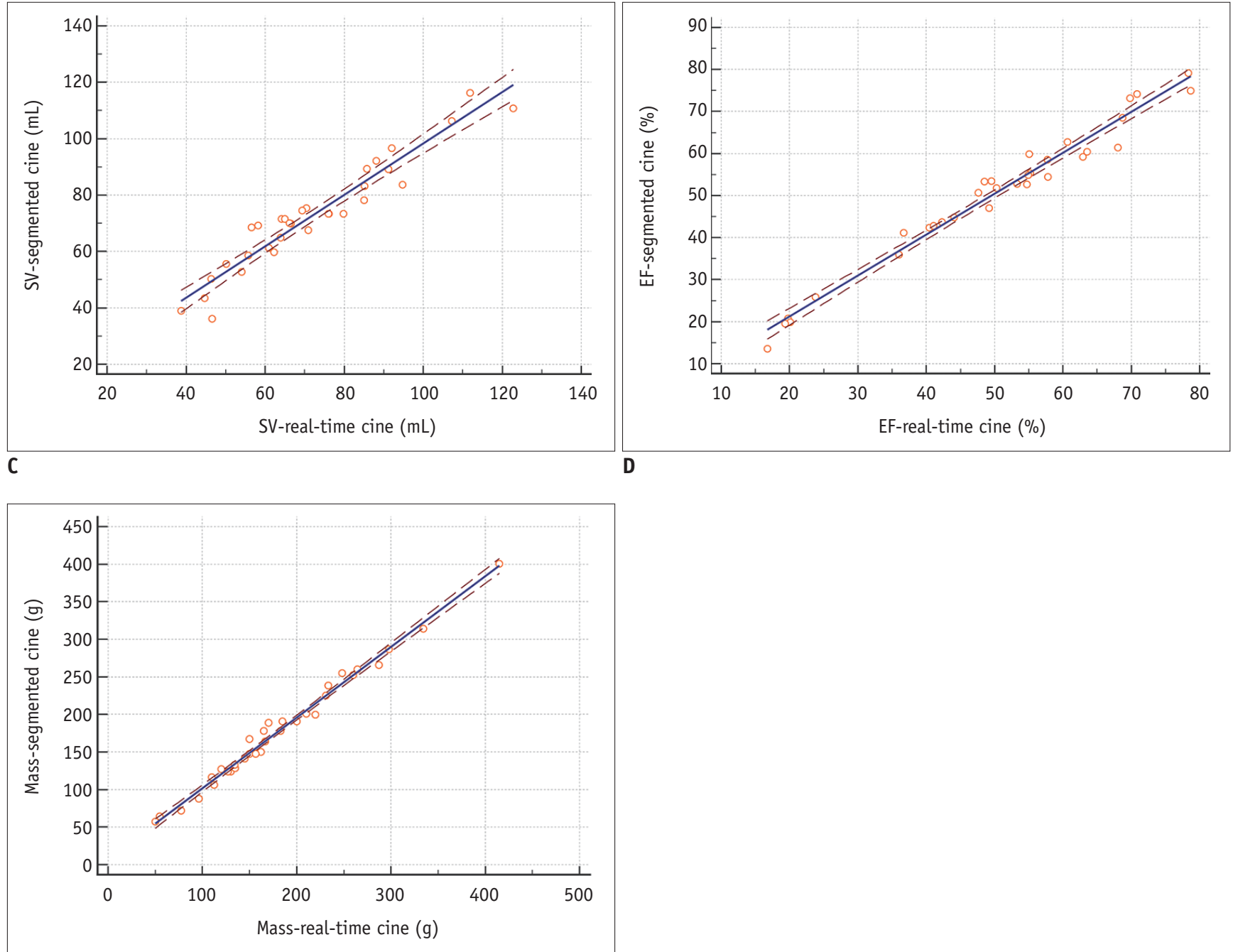

E

Fig. 6. Scatter plots for LV volumetric and mass measurements in standard segmented cine and real-time cine show good correlation in EDV (A), ESV (B), SV (C), EF (D), and mass (E) assessment. Brown dashed line indicates $95 \%$ confidence interval. 
and 70 ms respectively) (6). Consistent with the previously reported observations $(8,12)$, our results showed that cardiac cine with the temporal resolution of $60 \mathrm{~ms}$ is adequate for accurate assessment of ventricular functional values.

One strength of the current study was that two sets of image quality assessment criteria were used. EuroCMR score was applied in the Euro-CMR registry (24) and other CMR studies with emphasis on image quality (18). This score evaluates the image quality of cardiac cine in 12 aspects; however, it mainly focuses on the artifacts affecting the ventricular volume measurement rather than the visualization of cardiac structures. Therefore, we used another criterion to assess the performance of both sequences in structure visualization rated by radiologists. The real-time cine yielded slightly worse visualization of cardiac structures in group 1 because of limited specialtemporal resolution. On the contrary, it had lower EuroCMR score and a higher cardiac structure visualization rating in group 2 . This can be explained by several factors. Due to the segmented k-space sampling, the image quality of segmented cine strongly depends on the consistency of cardiac motion and is highly sensitive to irregular heartbeats aroused by either premature beats or atrial fibrillation. The data used to generate each slice in segmented cine was acquired from 6-12 heartbeats, while real-time cine images of each slice were obtained within one R-R interval. Therefore, the real-time cine is unlikely to be influenced by mis-triggering aroused by arrhythmia. Among patients with breath-holding difficulty, respiratory artifacts were found in 4 patients on segmented cine, which were less commonly observed on real-time cines because the acquisition time of each slice in real-time cine was short enough to eliminate the influence of involuntary respiratory motion. These results indicated that real-time cine may be a suboptimal solution for disease diagnosis, but it can provide images with adequate quality for volumetric measurement, particularly in patients with difficulty in breath-holding and severe arrhythmia.

More advanced acceleration techniques such as CS (10, $11,13,23)$ or sequences using radial sampling $(7,19)$ can increase the image acquisition speed significantly, which permits image acquisition covering the entire left ventricle within a single breath-hold with relatively high spatial and temporal resolution. Using these techniques, prospective triggered real-time imaging can be obtained. However, additional effort and time are required in image reconstruction $(10,13)$, and a significant underestimation of EDV was reported (13). The real-time cine in the current study was not implemented with these techniques; however, when combined with the CS technique, the spatial and temporal resolution of retrospective gated real-time cine could be improved in future work.

We acknowledge the following limitations in the present study. First, this is a single-center study with a limited sample size; however, it is comparable to previous studies that have attempted to validate the accuracy of volumetric assessment in the clinical setting $(7,11)$. Second, segmented cardiac cine was regarded as the gold standard for cardiac parameter assessment in cardiac MRI. However, in patients with severe artifacts that compromised parameter assessment (group 2), the reference standards of LV function and mass were unavailable. For this reason, we did not perform the parameter comparison and agreement analysis in these patients. Third, within our study cohort, the measured HRs were between 50 beats/min and 88 beats/min. In patients with a higher HR, the in-plane resolution will be lower, and the accuracy of this technique requires to be studied in the future.

In conclusion, real-time cine at $3 \mathrm{~T}$ allowed the assessment of LV volume and mass with high accuracy and good reproducibility in patients with normal breath-holding as well as patients with sinus rhythm. In patients with suboptimal breath-holding and/or arrhythmia, compared to the conventional segmented cine imaging, real-time cines showed better image quality and thus can be used as an alternative technique in this subgroup of patients.

\section{Conflicts of Interest}

The authors have no financial conflicts of interest.

ORCID

Shihua Zhao

https://orcid.org/0000-0003-1339-7476

Chen Cui

https://orcid.org/0000-0002-8996-6623

\section{REFERENCES}

1. Knauth AL, Gauvreau K, Powell AJ, Landzberg MJ, Walsh $E P$, Lock JE, et al. Ventricular size and function assessed by cardiac MRI predict major adverse clinical outcomes late after tetralogy of Fallot repair. Heart 2008;94:211-216

2. Curtis JP, Sokol SI, Wang Y, Rathore SS, Ko DT, Jadbabaie F, 
et al. The association of left ventricular ejection fraction, mortality, and cause of death in stable outpatients with heart failure. J Am Coll Cardiol 2003;42:736-742

3. Karamitsos TD, Francis JM, Myerson S, Selvanayagam JB, Neubauer $\mathrm{S}$. The role of cardiovascular magnetic resonance imaging in heart failure. J Am Coll Cardiol 2009;54:1407-1424

4. Moon JC, Lorenz CH, Francis JM, Smith GC, Pennell DJ. Breath-hold FLASH and FISP cardiovascular MR imaging: left ventricular volume differences and reproducibility. Radiology 2002;223:789-797

5. Kühl HP, Spuentrup E, Wall A, Franke A, Schröder J, Heussen $\mathrm{N}$, et al. Assessment of myocardial function with interactive non-breath-hold real-time MR imaging: comparison with echocardiography and breath-hold cine MR imaging. Radiology 2004;231:198-207

6. Hori Y, Yamada N, Higashi M, Hirai N, Nakatani S. Rapid evaluation of right and left ventricular function and mass using real-time true-FISP cine MR imaging without breathhold: comparison with segmented true-FISP cine MR imaging with breath-hold. J Cardiovasc Magn Reson 2003;5:439-450

7. Voit D, Zhang S, Unterberg-Buchwald C, Sohns JM, Lotz J, Frahm J. Real-time cardiovascular magnetic resonance at 1.5 T using balanced SSFP and 40 ms resolution. J Cardiovasc Magn Reson 2013;15:79

8. Kaji S, Yang PC, Kerr AB, Tang WH, Meyer $\mathrm{CH}$, Macovski A, et al. Rapid evaluation of left ventricular volume and mass without breath-holding using real-time interactive cardiac magnetic resonance imaging system. J Am Coll Cardiol 2001;38:527-533

9. Schwab F, Schwarz F, Dietrich 0, Lanz T, Resmer F, Wichmann $T$, et al. Free breathing real-time cardiac cine imaging with improved spatial resolution at 3 T. Invest Radiol 2013;48:158166

10. Sudarski S, Henzler T, Haubenreisser H, Dösch C, Zenge MO, Schmidt $M$, et al. Free-breathing sparse sampling cine MR imaging with iterative reconstruction for the assessment of left ventricular function and mass at 3.0 T. Radiology 2017;282:74-83

11. Vincenti G, Monney P, Chaptinel J, Rutz T, Coppo S, Zenge MO, et al. Compressed sensing single-breath-hold CMR for fast quantification of LV function, volumes, and mass. JACC Cardiovasc Imaging 2014; 7:882-892

12. Yamamuro M, Tadamura E, Kanao S, Okayama S, Okamoto J, Urayama $S$, et al. Cardiac functional analysis by free-breath real-time cine CMR with a spatiotemporal filtering method, TSENSE: comparison with breath-hold cine CMR. J Cardiovasc Magn Reson 2006;8:801-807
13. Feng L, Srichai MB, Lim RP, Harrison A, King W, Adluru G, et al. Highly accelerated real-time cardiac cine MRI using k-t SPARSE-SENSE. Magn Reson Med 2013;70:64-74

14. Xue H, Kellman P, Larocca G, Arai AE, Hansen MS. High spatial and temporal resolution retrospective cine cardiovascular magnetic resonance from shortened free breathing real-time acquisitions. J Cardiovasc Magn Reson 2013;15:102

15. Kellman P, Chefd'hotel C, Lorenz CH, Mancini C, Arai AE, McVeigh ER. Fully automatic, retrospective enhancement of real-time acquired cardiac cine MR images using image-based navigators and respiratory motion-corrected averaging. Magn Reson Med 2008;59:771-778

16. Sievers B, Kirchberg S, Bakan A, Franken U, Trappe HJ. Impact of papillary muscles in ventricular volume and ejection fraction assessment by cardiovascular magnetic resonance. $J$ Cardiovasc Magn Reson 2004;6:9-16

17. Klinke V, Muzzarelli S, Lauriers N, Locca D, Vincenti G, Monney $\mathrm{P}$, et al. Quality assessment of cardiovascular magnetic resonance in the setting of the European CMR registry: description and validation of standardized criteria. $J$ Cardiovasc Magn Reson 2013;15:55

18. Camargo GC, Erthal F, Sabioni L, Penna F, Strecker R, Schmidt $M$, et al. Real-time cardiac magnetic resonance cine imaging with sparse sampling and iterative reconstruction for leftventricular measures: comparison with gold-standard segmented steady-state free precession. Magn Reson Imaging 2017;38:138-144

19. Aandal G, Nadig V, Yeh V, Rajiah P, Jenkins T, Sattar A, et al. Evaluation of left ventricular ejection fraction using throughtime radial GRAPPA. J Cardiovasc Magn Reson 2014;16:79

20. Siontis KC, Geske JB, Ong K, Nishimura RA, Ommen SR, Gersh BJ. Atrial fibrillation in hypertrophic cardiomyopathy: prevalence, clinical correlations, and mortality in a large high-risk population. J Am Heart Assoc 2014;3:e001002

21. Ceresne L, Upshur RE. Atrial fibrillation in a primary care practice: prevalence and management. BMC Fam Pract 2002;3:11

22. Braunwald E. Heart failure. JACC Heart Fail 2013;1:1-20

23. Kido T, Kido T, Nakamura M, Watanabe K, Schmidt M, Forman $C$, et al. Compressed sensing real-time cine cardiovascular magnetic resonance: accurate assessment of left ventricular function in a single-breath-hold. J Cardiovasc Magn Reson 2016;18:50

24. Bruder 0 , Wagner A, Lombardi M, Schwitter J, van Rossum A, Pilz G, et al. European cardiovascular magnetic resonance (EuroCMR) registry--multi-national results from 57 centers in 15 countries. J Cardiovasc Magn Reson 2013;15:9 